Article

\title{
Frequency-Dependent Magnetic Susceptibility of Magnetite and Cobalt Ferrite Nanoparticles Embedded in PAA Hydrogel
}

\author{
Susanne van Berkum, Joris T. Dee, Albert P. Philipse and Ben H. Erné * \\ Van 't Hoff Laboratory for Physical and Colloid Chemistry, Debye Institute for Nanomaterials Science, \\ Utrecht University, Padualaan 8, 3584 CH Utrecht, The Netherlands; \\ E-Mails: s.vanberkum@uu.nl (S.B.); joris.dee@gmail.com (J.T.D.); a.p.philipse@uu.nl (A.P.P.) \\ * Author to whom correspondence should be addressed; E-Mail: b.h.erne@uu.nl; \\ Tel.: +31-30-253-2934; Fax: +31-30-253-3870.
}

Received: 11 April 2013; in revised form: 29 April 2013 / Accepted: 7 May 2013 /

Published: 14 May 2013

\begin{abstract}
Chemically responsive hydrogels with embedded magnetic nanoparticles are of interest for biosensors that magnetically detect chemical changes. A crucial point is the irreversible linkage of nanoparticles to the hydrogel network, preventing loss of nanoparticles upon repeated swelling and shrinking of the gel. Here, acrylic acid monomers are adsorbed onto ferrite nanoparticles, which subsequently participate in polymerization during synthesis of poly(acrylic acid)-based hydrogels (PAA). To demonstrate the fixation of the nanoparticles to the polymer, our original approach is to measure low-field AC magnetic susceptibility spectra in the $0.1 \mathrm{~Hz}$ to $1 \mathrm{MHz}$ range. In the hydrogel, the magnetization dynamics of small iron oxide nanoparticles are comparable to those of the particles dispersed in a liquid, due to fast Néel relaxation inside the particles; this renders the ferrogel useful for chemical sensing at frequencies of several kHz. However, ferrogels holding thermally blocked iron oxide or cobalt ferrite nanoparticles show significant decrease of the magnetic susceptibility resulting from a frozen magnetic structure. This confirms that the nanoparticles are unable to rotate thermally inside the hydrogel, in agreement with their irreversible fixation to the polymer network.
\end{abstract}

Keywords: ferrohydrogel; hydrogel; ferrogel; poly(acrylic acid); iron oxide; cobalt ferrite; magnetic nanoparticles; magnetic susceptibility; Brownian relaxation; Néel relaxation 


\section{Introduction}

Hydrogels are important for biomedical applications, for instance in drug delivery [1-3] and biosensors $[4,5]$. They are highly cross-linked networks of polymer strands that can increase their volume by uptake of water without changing their shape or structure [1]. Hydrogels with acidic groups like poly(acrylic acid) (PAA) swell with increasing $\mathrm{pH}$ due to increasing repulsive forces of ionized groups [6] and hydrogels may also respond to other factors, such as changes in temperature, ionic strength or other stimuli [1]. This makes hydrogels interesting for biosensor applications. In 2010, van Bruggen and van Zon [7] reported on the feasibility of an in vivo biosensor composed of a $\mathrm{pH}$ responsive hydrogel containing superparamagnetic nanoparticles on top of a GMR (giant magneto-resistive) sensor. Nanoparticles were magnetically aligned by a high frequency field generated by excitation wires and the field of the nanoparticles was measured by the GMR sensor using a lock-in amplifier. Although the authors were not able to prepare a suitable ferrogel and tested their prototype sensor with a ferrofluid, in principle, the functionality of the sensor is based on the swelling and shrinking of the hydrogel in which the nanoparticles are dispersed. Upon swelling due to an increase of $\mathrm{pH}$, the field of the nanoparticles at the GMR sensor decreases, whereas it increases upon shrinking. In this way, change in $\mathrm{pH}$ is indirectly measured by a change in magnetic signal. Here, we investigate the preparation of hydrogels incorporating magnetic nanoparticles and we demonstrate chemical fixation to the polymer network by measuring the frequency-dependent AC magnetic susceptibility.

The chemical binding of nanoparticles to a hydrogel is achieved by adsorbing polymerizable molecules on the surface of the nanoparticles such that the nanoparticles are not only incorporated in the hydrogel but also become part of the polymer network [8]. Messing et al. [9] reported on the transfer of $\mathrm{CoFe}_{2} \mathrm{O}_{4}$ nanoparticles from nonpolar to aqueous media, while at the same time, the surface was functionalized so that the nanoparticles functioned as cross-linkers in a poly(acryl amide) gel. Establishing the function of the nanoparticles as cross-linkers was demonstrated by leakage experiments and by dissolution of the particles upon which the hydrogel fully disintegrated. A similar transfer was described by Vo et al. [10] by exchanging oleic acid molecules on $\mathrm{TiO}_{2}$ nanoparticles with acrylic acid. In our case, we use adsorbed acrylic acid, to fix nanoparticles to a PAA network. We first positively charge the nanoparticles via a treatment with nitric acid. The first reason for this is to prevent incorporation of large aggregates of particles into the polymer network: positively and negatively charged particles remain well dispersed in aqueous dispersions for long times, but nanoparticles with a negative charge at neutral or high $\mathrm{pH}$ immediately form large aggregates upon mixing with the acidic monomer solution, whereas positively charged particles do not. The second reason for giving our nanoparticles initially a positive charge is that spontaneous adsorption of the carboxylic acid group of acrylic acid to the surface of the nanoparticles is facilitated. The end result is that the double bond of the adsorbed acrylic acid points outwards so that it can participate in polymerization, yielding a visually homogeneous gel (Figure 1). 
Figure 1. (left) Schematic illustration of the surface treatments of our nanoparticles; (right) a magnetite (S) dispersion and a hydrogel with incorporated magnetite (S).
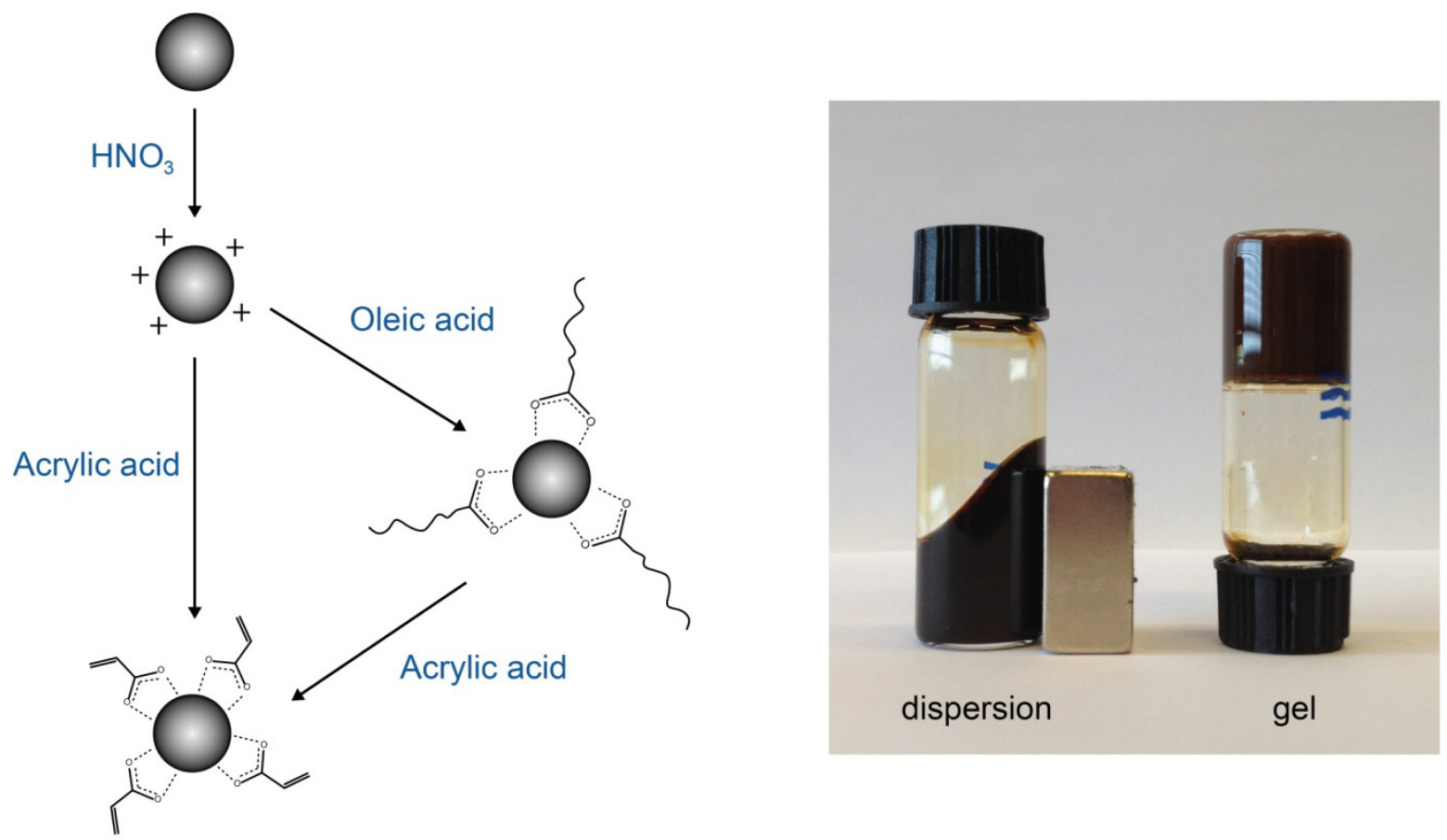

To investigate whether the particles coated with acrylic acid are bound to the hydrogel, our original approach is to examine rotational diffusion by measuring low-field frequency-dependent AC susceptibility spectra in the low-field limit. This technique is known for instance from bio-essays that have been developed to detect magnetically the slowdown of rotational diffusion when molecules absorb to the particle surface, enlarging the hydrodynamic radius [11-15]; it has also been used to monitor the gelation of aqueous gelatin at the scale of magnetic probe nanoparticles [16]. The dimensionless magnetic susceptibility $\chi$ gives the magnetization $(M)$ in $A / m$ per applied external field $(\mathrm{H})$ in $\mathrm{A} / \mathrm{m}$. When the external magnetic field alternates, the particle magnetic moments of nanoparticles dispersed in a carrier liquid can reorient through two different mechanisms: Brownian relaxation and Néel relaxation [17,18]. Brownian relaxation is the only option for nanoparticles whose magnetic moment is blocked inside the particle: they must physically rotate in the solvent. The characteristic frequency of Brownian relaxation is given by

$$
2 \pi f_{B}=\omega_{B}=2 D_{r}=k_{B} T /\left(4 \pi \eta a_{h}^{3}\right)
$$

where $f_{\mathrm{B}}$ is the Brownian relaxation frequency in $\mathrm{Hz}, \omega_{\mathrm{B}}$ the radial frequency in $\mathrm{s}^{-1}, D_{\mathrm{r}}$ the rotational diffusion coefficient, $k_{\mathrm{B}} T$ the thermal energy, $\eta$ the viscosity of the solvent, and $a_{\mathrm{h}}$ the hydrodynamic radius of the nanoparticles. For smaller magnetic nanoparticles, the magnetic moment can rotate fast within the particle, with a characteristic frequency of Néel relaxation given by the following expression,

$$
2 \pi f_{N}=\omega_{N}=2 \pi f_{0} \exp \left[-\frac{K V}{k_{B} T}\right]
$$

where $f_{\mathrm{N}}$ is the Néel relaxation frequency in $\mathrm{Hz}, \omega_{\mathrm{N}}$ the radial frequency in $\mathrm{s}^{-1}, f_{0}$ the Larmor frequency, $K$ the magnetic anisotropy constant, and $V$ the magnetic core volume. The frequency dependent magnetic susceptibility has a real and an imaginary part: $\chi=\chi^{\prime}-i \chi^{\prime \prime}$. The real part $\chi^{\prime}$ has a 
plateau in the low frequency regime and equals the initial magnetic susceptibility from a magnetization curve without hysteresis $(\mathrm{M}$ versus $\mathrm{H})$. As the frequency of the alternating field increases, the magnetization of the particles is not able to keep up with the alternating field and the $\chi^{\prime}$ decreases. The imaginary part $\chi^{\prime \prime}$ of the magnetic susceptibility shows a maximum at the characteristic relaxation frequency.

The biosensor concept of van Bruggen and van Zon [7] requires a superparamagnetic gel, with magnetic particles that can respond to high operational frequency. Here, we will prepare ferrogels using not only magnetic nanoparticles with fast Néel relaxation, but also with particles having thermally blocked particle magnetic moments. In the latter case, any magnetic susceptibility signal indicates that the particles are physically moving and not well fixed inside the hydrogel (Figure 2). Three types of nanoparticles will be incorporated into gels: superparamagnetic iron oxide nanoparticles (S), larger iron oxide nanoparticles (L) with the same surface chemistry but much longer Néel relaxation, and cobalt ferrite nanoparticles with even longer Néel relaxation due to their high magnetic anisotropy [19].

Figure 2. Schematic representation of nanoparticles with different relaxation mechanisms. Particles that are not part of the hydrogel network should be able to rotate whereas particles that are bound to the hydrogel network will not show any magnetic relaxation when the particle magnetic moment is blocked.

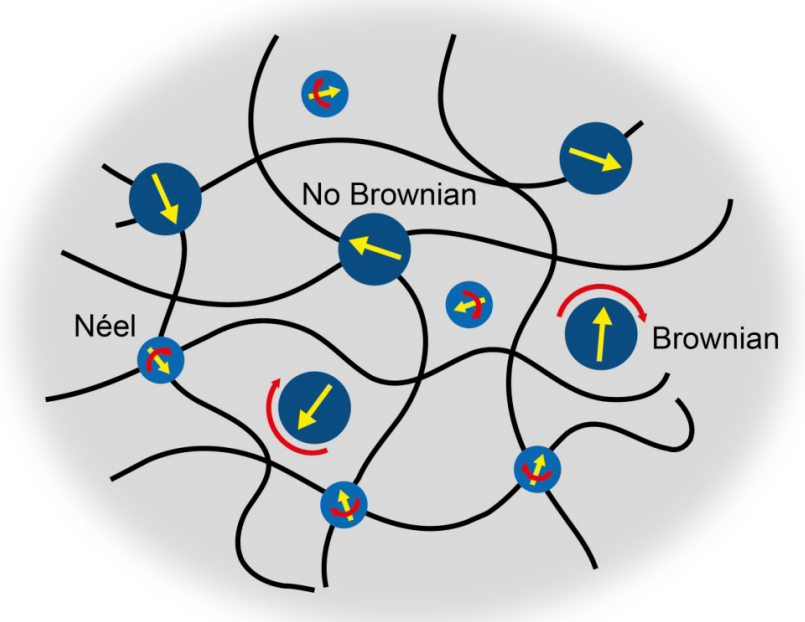

The following section first describes the magnetic nanoparticles and their surface modification. This is followed by a discussion of fixation of the nanoparticles in the hydrogels as investigated by their frequency-dependent AC magnetic susceptibility.

\section{Results and Discussion}

\subsection{Characterization of the Magnetic Nanoparticles}

TEM pictures of the three types of synthesized magnetic nanoparticles are shown in Figure 3 together with corresponding size distributions. Due to the aqueous precipitation methods, the size distributions are relatively broad, with polydispersities on the order of $30 \%$. From the black color of 
the iron oxide synthesis products, it was concluded that they were mainly magnetite as expected from the synthesis methods.

Figure 3. Transmission electron microscopy images of the synthesized nanoparticles with corresponding size distributions: (a) magnetite (S), $d \sim 9 \mathrm{~nm},($ b) magnetite (L), $d \sim 23 \mathrm{~nm}$, (c) cobalt ferrite, $d \sim 22 \mathrm{~nm}$.

(a)
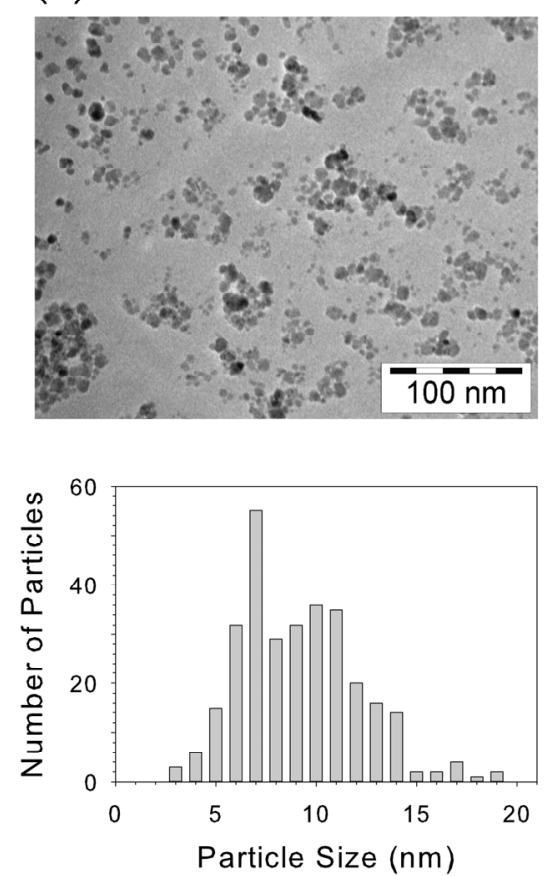

(b)
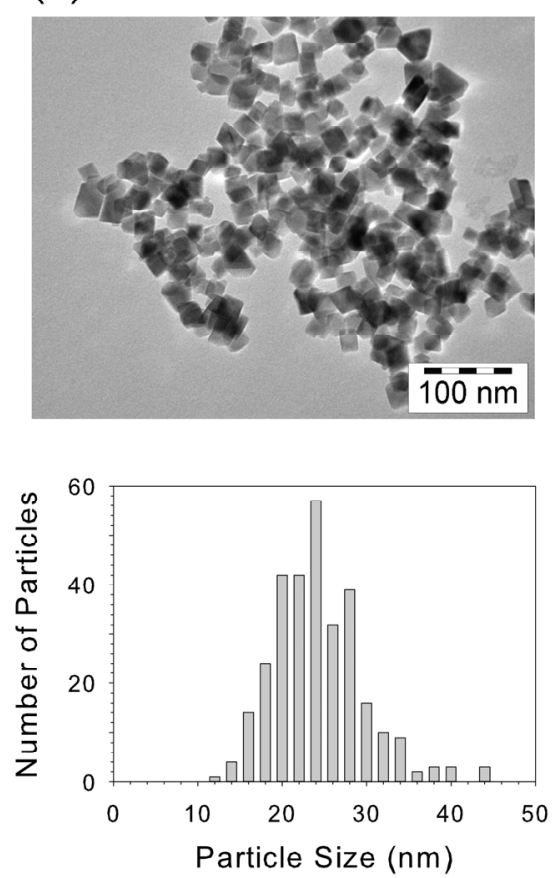

(c)
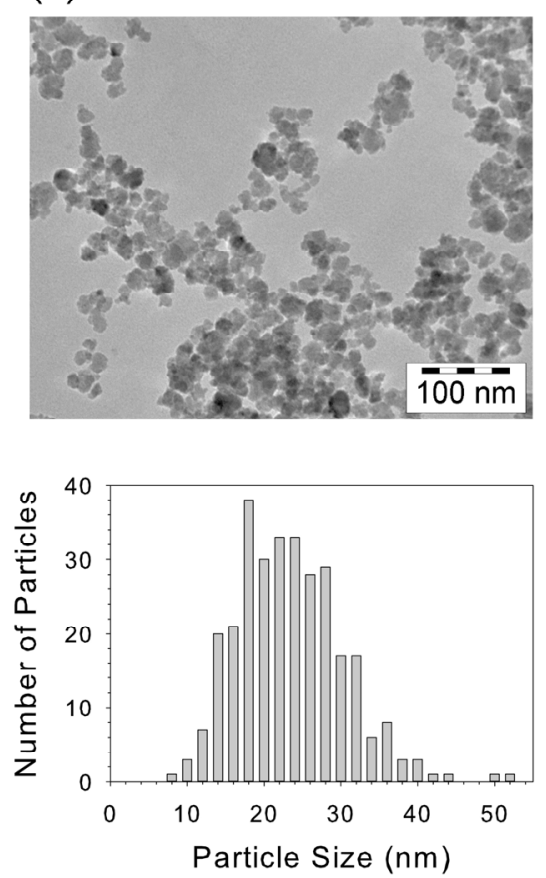

Differences in magnetization dynamics are already visible in the magnetization curves of the dry particles in Figure 4. The slower the magnetic relaxation, the higher the ratio between the remanent magnetization, $M_{\mathrm{R}}$, and the saturation magnetization, $M_{\mathrm{S}}$. The magnetic properties are summarized in Table 1. The values for $M_{\mathrm{S}}$ are lower than those of bulk magnetite, $92 \mathrm{emu} / \mathrm{g}$ [20], which is ascribed to nanoparticle surface effects [21-26]. These effects also occur for the cobalt ferrite nanoparticles, whose saturation magnetization is also lower than the bulk value of $\sim 80 \mathrm{emu} / \mathrm{g}$. The magnetization curve in Figure 4a shows that the sample with magnetite $(\mathrm{S})$ has no remanent magnetization, due to rapid Néel relaxation. The magnetite (L) and cobalt ferrite samples, however, show $M_{\mathrm{R}} / M_{\mathrm{S}}$ values of 0.19 and 0.34 respectively, compared to $M_{\mathrm{R}} / M_{\mathrm{S}}=0.5$ for completely blocked noninteracting nanoparticles with random orientation of the easy axis The last column of Table 1 gives an estimate of the dipolar contact interaction of the nanoparticles,

$$
\lambda=\frac{\mu_{0} \mu^{2}}{4 \pi k_{B} T d^{3}}
$$

where $\mu_{0}$ is the permeability of vacuum, $\mu$ is the magnetic dipole moment of a nanoparticle $\left(\mu=\frac{\pi d^{3} M_{S}}{6}\right)$, and $d$ is the particle diameter. 
Figure 4. Magnetization curves of the dry nanoparticles at room temperature: (a) magnetite (S), (b) magnetite (L), and (c) cobalt ferrite.
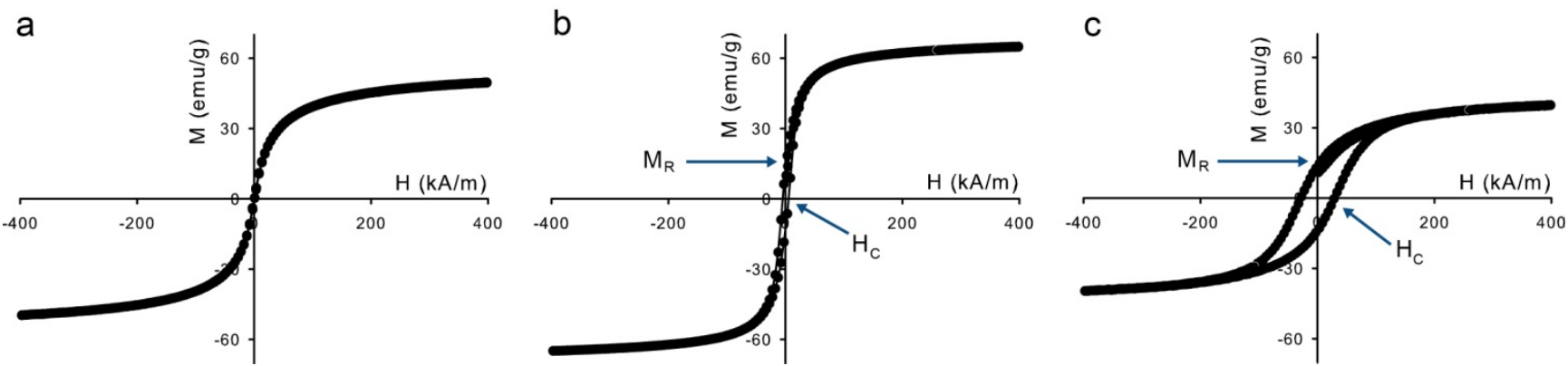

Table 1. Magnetic properties of the nanoparticles. The standard deviations given in parentheses are from performing triplicate analyses.

\begin{tabular}{lcccc}
\hline & $\boldsymbol{M}_{\mathbf{S}}(\mathbf{e m u} / \mathbf{g})$ & $\boldsymbol{M}_{\mathbf{R}} / \boldsymbol{M}_{\mathbf{S}}$ & $\boldsymbol{H}_{\mathbf{C}}(\mathbf{k A} / \mathbf{m})$ & $\boldsymbol{\lambda}$ \\
\hline Magnetite (S) & $54.8(1.2)$ & $0.007(0.001)$ & - & 0.4 \\
Magnetite (L) & $67.5(0.3)$ & $0.187(0.003)$ & $5.09(0.05)$ & 10 \\
Cobalt ferrite & $38(4)$ & $0.343(0.013)$ & $34(4)$ & 3 \\
\hline
\end{tabular}

\subsection{Surface Modification of Magnetite Nanoparticles Using Acrylic Acid}

The infrared spectrum of nitric acid-treated magnetite (S) shows a strong peak of asymmetric stretch of $\mathrm{NO}_{3}^{-}$at $1384 \mathrm{~cm}^{-1}$ (Figure 5). Magnetite (L) and cobalt ferrite do not show such a strong peak due to their lower surface-to-volume ratio. The spectra of magnetite (S) and cobalt ferrite both show the presence of water by a broad band around $3400 \mathrm{~cm}^{-1}$ and a peak at $1600 \mathrm{~cm}^{-1}$. Magnetite (S) shows maghemite features at $630 \mathrm{~cm}^{-1}$ [27] caused by the heat treatment during $\mathrm{KBr}$ sample preparation. Similar features are also observed for magnetite (L) but due to the larger size of the particles, oxidation only affects the surface of the particles and magnetite dominates with its absorbance at $560 \mathrm{~cm}^{-1}$. The spectrum for cobalt ferrite displays stretching vibrations of $\mathrm{Fe}^{3+}-\mathrm{O}^{2-}$ in the tetrahedral sites at $574 \mathrm{~cm}^{-1}$ [28]. The band around $870 \mathrm{~cm}^{-1}$ does not originate from cobalt ferrite [28] or cobalt oxide [29], but we suspect it corresponds to an iron hydroxide phase [30].

Figure 5. IR spectra of the positively charged nanoparticles: (a) magnetite (S), (b) magnetite (L), and (c) cobalt ferrite.

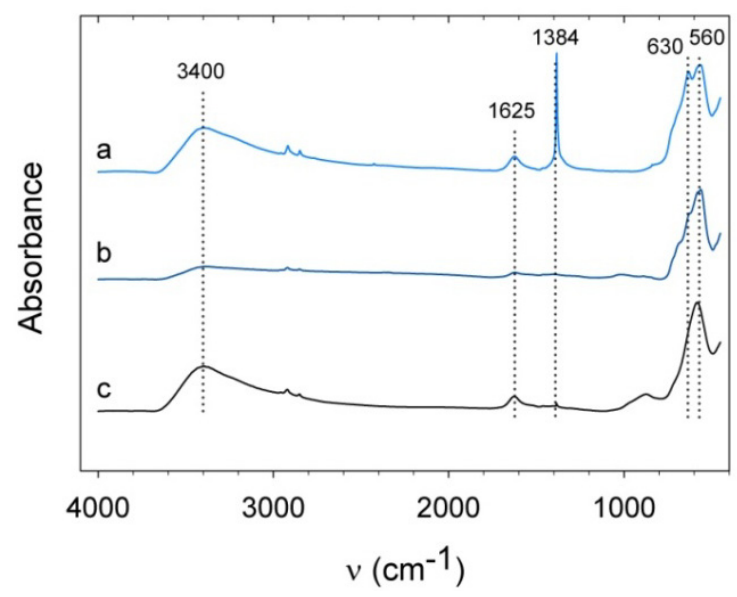


To fix the nanoparticles to the hydrogel network, surface modification with acrylic acid [10] was performed next. Figure 6 shows the infrared spectra of nanoparticles that were mixed for two minutes with an acrylic acid/water mixture and for comparison the spectrum of acrylic acid. The latter shows characteristic features of acrylic acid: a $\mathrm{C}=\mathrm{O}$ stretch at $1695 \mathrm{~cm}^{-1}$, an $\mathrm{C}=\mathrm{C}$ stretch at $1634 \mathrm{~cm}^{-1}$, an asymmetric stretch of $\mathrm{CO}_{2}^{-}$at $1614 \mathrm{~cm}^{-1}$, and a symmetric stretch of $\mathrm{CO}_{2}^{-}$at $1431 \mathrm{~cm}^{-1}[31,32]$. The peaks between 1200 and $1300 \mathrm{~cm}^{-1}$ can be ascribed to $\mathrm{C}-\mathrm{OH}$ stretch and the weak absorbances between 800 and $1050 \mathrm{~cm}^{-1}$ originate from $\mathrm{CH}$ out of plane bending [33]. The peak for the $\mathrm{C}=\mathrm{C}$ stretch at $1634 \mathrm{~cm}^{-1}$ slightly shifts to $1639 \mathrm{~cm}^{-1}$ when adsorbed onto the nanoparticles surface. The sharp peak at $1695 \mathrm{~cm}^{-1}$ disappears for acrylic acid adsorbed onto nanoparticles, whereas the symmetric stretch of $\mathrm{CO}_{2}^{-}$is enhanced and shifts to $1438 \mathrm{~cm}^{-1}$, pertaining to an adsorbed carboxylate group [32]. This is valid for both magnetite (S) and cobalt ferrite. The surface group bands for magnetite (L) are much weaker due to the lower surface-to-volume ratio of the larger particles.

Figure 6. IR spectra of (a) pure acrylic acid and acrylic acid coated nanoparticles: (b) magnetite (S), (c) magnetite (L), and (d) cobalt ferrite.

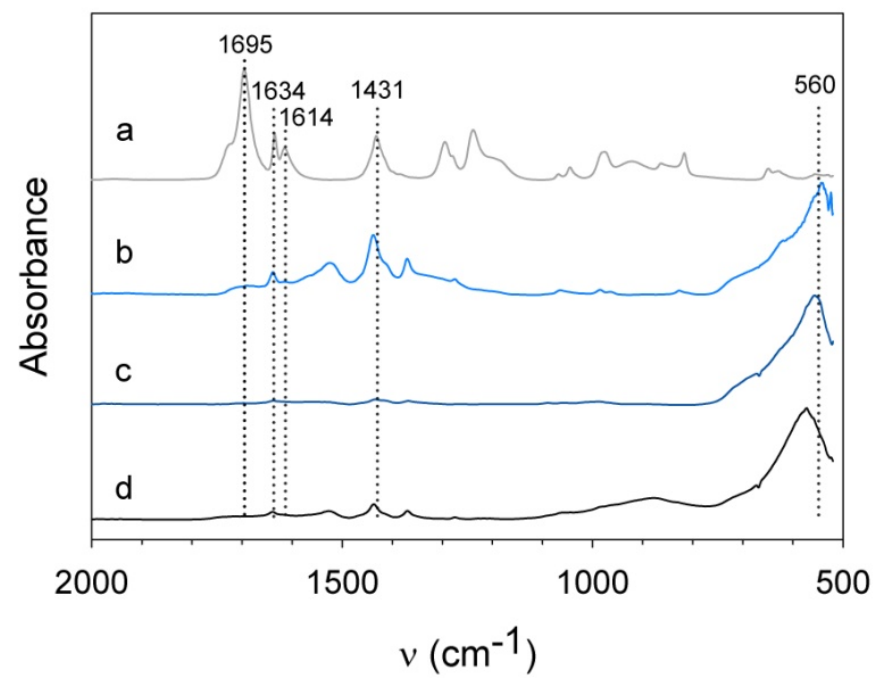

We discovered that treatment time of the acrylic acid surface modification method must be kept limited to prevent dissolution of the particles, especially when they are not initially covered with other adsorbed molecules. When the procedure was performed with bare particles overnight as was done with oleic acid coated particles by Vo et al. [10], the color of the dispersion changed orange and the magnetic susceptibility decreased dramatically. Magnetic susceptibility measurements at $1 \mathrm{~Hz}$ show that the magnetic susceptibility for positively charged iron oxide nanoparticles decreased to the noise level within $40 \mathrm{~min}$ (Figure 7). Oleic acid coated nanoparticles, however, required $10 \mathrm{~h}$ for a similar decrease, indicating that dissolution is slowed down because ligand exchange must occur. Figure 7 demonstrates that spontaneous adsorption of acrylic acid onto the surface of iron oxide NPs is rapid; therefore mixing of positively charged iron oxide NPs with the monomer mixture for two minutes should be sufficient for adsorption of acrylic acid. 
Figure 7. Low-field magnetic susceptibility (real susceptibility at $1 \mathrm{~Hz}$ ) of magnetite (S) with a positively charged bare surface and oleic acid coated magnetite $(\mathrm{S})$ dispersed in $90 \mathrm{vol} \%$ solution of acrylic acid in water, normalized to their initial value $\chi_{\max }$ at $1 \mathrm{~Hz}$. Positively charged magnetite (S) shows an immediate decrease of magnetic susceptibility due to dissolution of the particles, whereas the decrease of the magnetic susceptibility of oleic acid coated nanoparticles is slowed down by ligand exchange.

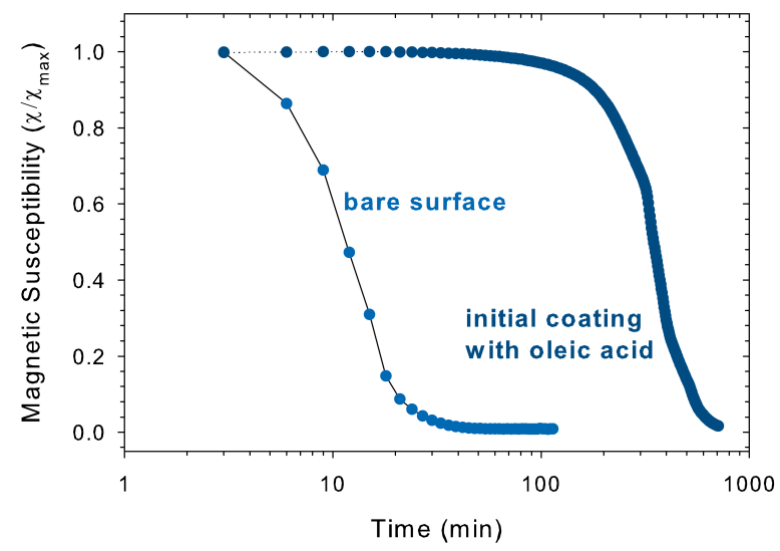

\subsection{Magnetic Relaxation of Ferrogels}

For the hydrogels with embedded nanoparticles, magnetization curves are shown in Figure 8 . The magnetic properties are summarized in Table 2. The saturation magnetization of each sample is in the same order as expected from the volume fraction of magnetic material present. Striking is that the relative remanence $M_{\mathrm{R}} / M_{\mathrm{S}}$ and coercive field of magnetite (L) are increased by $27 \%$ and $95 \%$ respectively compared to the values in Table 1. This effect is ascribed to changes in the magnetic interactions between the nanoparticles within the gel. Dipolar structures are formed when the contact interaction of magnetic nanoparticles is sufficiently high compared to the thermal energy $\mathrm{k}_{\mathrm{B}} \mathrm{T}[34,35]$ which is the case for magnetite (L) and cobalt ferrite, see Table 1 , where the $\lambda$ is the highest for magnetite (L) due to its large particle size. Introducing magnetic interactions locks the orientation of the magnetic dipoles inside the structures, which slows down the magnetization dynamics [36]. Evidence for particle interactions is given by the characteristic relaxation frequency of magnetite (L) and cobalt ferrite in dispersion shown in Figure 9, which reveals the presence of clusters, confirming that the particles are close enough for particle-particle interaction. For cobalt ferrite nanoparticles, a similar increase in $M_{\mathrm{R}} / M_{\mathrm{S}}$ is observed, although the coercive field has decreased slightly.

Figure 8. Magnetization curves of (a) magnetite (S), (b) magnetite (L), and (c) cobalt ferrite nanoparticles embedded in a hydrogel.
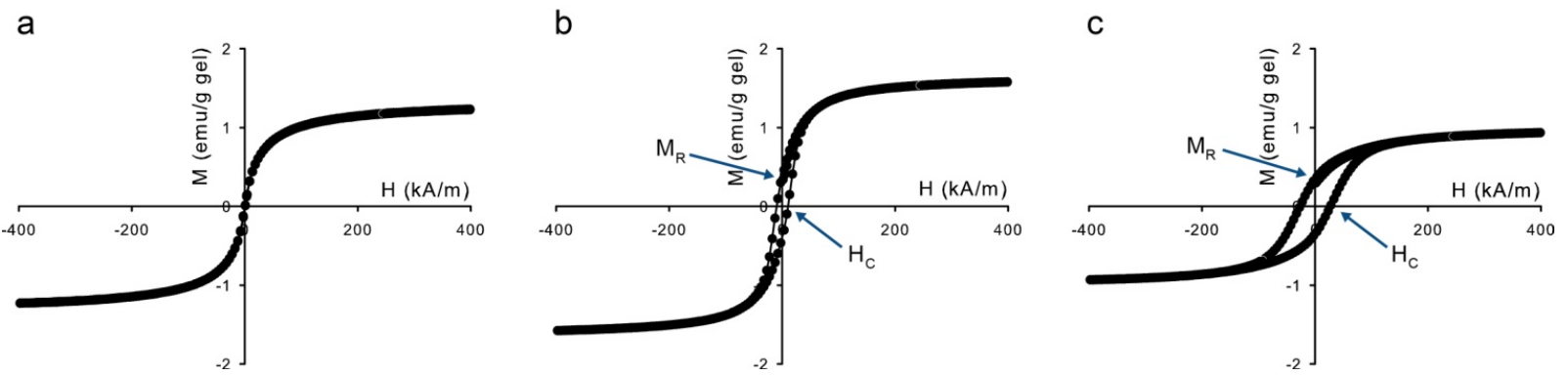
Table 2. Magnetic properties of the ferrogels. The standard deviations are given in parentheses from performing triplicate analyses.

\begin{tabular}{lccc}
\hline & $\boldsymbol{M}_{\mathbf{S}}($ emu per g gel) & $\boldsymbol{M}_{\mathbf{R}} / \mathbf{M}_{\mathbf{S}}$ & $\boldsymbol{H}_{\mathbf{C}}(\mathbf{k A} / \mathbf{m})$ \\
\hline Magnetite (S) & $1.20(0.06)$ & $0.009(0.000)$ & - \\
Magnetite (L) & $1.63(0.04)$ & $0.238(0.006)$ & $9.91(0.15)$ \\
Cobalt ferrite & $0.95(0.10)$ & $0.375(0.012)$ & $26.8(0.4)$ \\
\hline
\end{tabular}

Figure 9 shows magnetic susceptibility spectra for all three types of magnetic nanoparticles used in this study. The nanoparticles were measured both in dispersion and in hydrogel to investigate the difference in magnetic susceptibility between bound particles and particles that have a positively charged surface and are free to rotate in dispersion.

The characteristic relaxation frequency for magnetite $(S)$ differs when the particles are free in dispersion or embedded in a hydrogel. This can be explained by the wide size distribution and the effective relaxation time, $\tau_{\text {eff, }}$

$$
\frac{1}{\tau_{e f f}}=\frac{1}{\tau_{N}}+\frac{1}{\tau_{B}}
$$

where $\tau_{\mathrm{N}}$ is the Néel relaxation time and $\tau_{\mathrm{B}}$ is the Brownian relaxation time. The magnetic moments of the particles in dispersion are all free to rotate by a combination of both mechanisms, whereas this is not the case for particles in a hydrogel, for which Néel relaxation is the only remaining option. This is clearly the case in Figure 9a: in liquid dispersion, the magnetic moments of the particles are all free to rotate and $\tau_{\text {eff }}$ results from both relaxation mechanisms, whereas in the hydrogel network, Brownian relaxation is excluded; about $10 \%$ of the magnetic moments can no longer rotate since the particles are so large that their Néel relaxation is too slow. This leads to a shift in the characteristic relaxation frequency, since the effective relaxation time is now a result of only the small particles with fast Néel relaxation.

The imaginary part of the spectrum of the magnetite (L) nanoparticles in dispersion has a maximum at $8 \mathrm{~Hz}$ in Figure 9b, corresponding to clusters of about $400 \mathrm{~nm}$. This is in good agreement with the observation that the sample showed sediment after one day. The characteristic relaxation frequency of the cobalt ferrite nanoparticles is $800 \mathrm{~Hz}$ in liquid dispersion in Figure 9c, corresponding to clusters of about $80 \mathrm{~nm}$. In a carrier liquid the thermally blocked magnetite (L) and cobalt ferrite nanoparticles will relax by the Brownian mechanism [17,34-36], resulting in a strong decrease in magnetic susceptibility signal for nanoparticles immobilized in the hydrogel network, comparable to what happens when a ferrofluid is frozen [37]. The large decrease in magnetic susceptibility of the nanoparticles when incorporated in hydrogel means that the particles are no longer able to rotate.

The irreversible fixation of the nanoparticles to the polymer network was expected from the followed chemical methods, where the nanoparticles acted as cross-linkers (Figure 1). Contrary to [8,9] the nanoparticles in our case were not the sole cross-linkers, but by measuring the magnetic susceptibility, we could single out the motion of the nanoparticles and conclude that they were fixed in the gels. No leakage of nanoparticles was observed by us upon swelling and shrinking of the ferrogels, in contrast to studies on ferrogels in which part of the particles was still free to rotate $[38,39]$. For application in biosensors of the type described by van Bruggen en van Zon [7], our ferrogel with small 
magnetite particles (S) seems highly suitable: the particles are fixed to the polymer network and they respond strongly to alternating magnetic fields, at least up to an operational frequency of $50 \mathrm{kHz}$.

Figure 9. Frequency-dependent AC susceptibility spectra of liquid dispersions ("liquid") and poly(acrylic acid) hydrogels ("gel") incorporating: (a) $1.6 \mathrm{wt} \%$ of magnetite (S) nanoparticles, (b) $2 \mathrm{wt} \%$ of magnetite (L) nanoparticles, and (c) $2 \mathrm{wt} \%$ of cobalt ferrite nanoparticles. After immobilization of the nanoparticles in a gel, the rapid Néel relaxation of the small particles continues (a), except for a small fraction of larger particles. The magnetic relaxation of the larger magnetite particles (b) and cobalt ferrite (c) is almost fully quenched after incorporation in hydrogel because no Néel relaxation occurs and the particles are immobilized inside the polymer network.

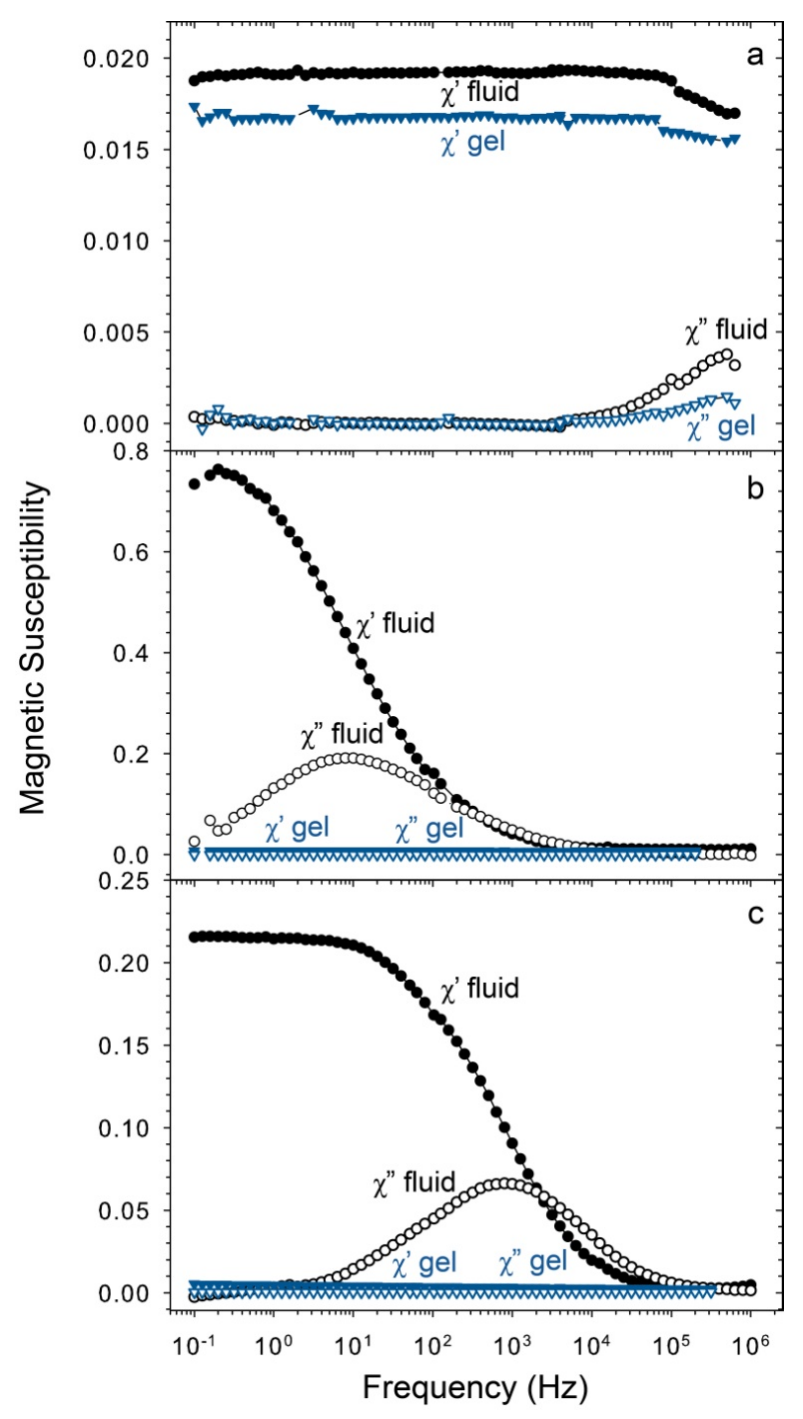

\section{Experimental Section}

\subsection{Materials}

Iron (II) chloride tetrahydrate $\left(\mathrm{FeCl}_{2} \cdot 4 \mathrm{H}_{2} \mathrm{O},>98 \%\right)$, iron (II) chloride hexahydrate $\left(\mathrm{FeCl}_{3} \cdot 6 \mathrm{H}_{2} \mathrm{O}\right.$, 97\%), and iron sulfate heptahydrate $\left(\mathrm{FeSO}_{4} \cdot 7 \mathrm{H}_{2} \mathrm{O}, 99 \%\right)$ were purchased from Sigma. Acrylic acid (AA, 99\% anhydrous), diethyleneglycol diacrylate (DEGDA, 75\%), and oleic acid (OA, techn. 90\%) 
were obtained from Aldrich. Cobalt (II) chloride hexahydrate $\left(\mathrm{CoCl}_{2} \cdot 6 \mathrm{H}_{2} \mathrm{O},>98 \%\right)$ was purchased from Fluka. Ethanol (EtOH, p.a.), n-hexane (p.a.), potassium nitrate $\left(\mathrm{KNO}_{3}, 99 \%\right)$, and sodium hydroxide $(\mathrm{NaOH}, 99 \%)$ were purchased from Merck (Darmstadt, Germany). Ammonia solution $\left(\mathrm{NH}_{4} \mathrm{OH}, 28 \mathrm{wt} \%-30 \mathrm{wt} \%\right.$ in water), sulfuric acid $\left(\mathrm{H}_{2} \mathrm{SO}_{4}, 96 \%\right)$, hydroxylethyl acrylate (HEA, 97\% stabilized), and 2,2'-azobis(2-methylpropionamidine) dihydrochloride (V-50, 98\%) were obtained from Acros Organics (Geel, Belgium). Toluene (techn.) was purchased from Interchema. All chemicals were used as received. Deionized water purified by a Millipore purification system (MILLIPORE Synergy 185) was used in all syntheses and washing steps.

\subsection{Preparation of Magnetic Nanoparticles}

Magnetite (S) nanoparticles - Small magnetite nanoparticles were prepared following the method reported by Massart et al. [40]: $3.93 \mathrm{~g} \mathrm{(20} \mathrm{mmol)} \mathrm{FeCl}_{2} \cdot 4 \mathrm{H}_{2} \mathrm{O}$ was dissolved in $10 \mathrm{~mL}$ of $2 \mathrm{M} \mathrm{HCl}$ and added to $10.89 \mathrm{~g} \mathrm{(40} \mathrm{mmol)} \mathrm{FeCl}_{3} \cdot 6 \mathrm{H}_{2} \mathrm{O}$ dissolved in $40 \mathrm{~mL}$ of water. The mixed solution was added rapidly to $500 \mathrm{~mL}$ of $0.7 \mathrm{M}$ ammonia solution under vigorous stirring to form a black precipitate. The mixture was stirred vigorously for another $25 \mathrm{~min}$ and subsequently sonicated for $5 \mathrm{~min}$. The particles were washed three times by magnetic sedimentation and redispersion in water. The particles were finally redispersed in water.

Magnetite (L) nanoparticles - Large magnetite nanoparticles were prepared as described by Andres Vergés et al. [41]: $0.56 \mathrm{~g} \mathrm{NaOH}(0.072 \mathrm{M})$ and $1.05 \mathrm{~g} \mathrm{KNO}_{3}(0.052 \mathrm{M})$ were dissolved in $80 \mathrm{~mL}$ of water and $100 \mathrm{~mL}$ of ethanol, forming solution A. For solution $\mathrm{B}, 56.4 \mu \mathrm{L}$ of concentrated $\mathrm{H}_{2} \mathrm{SO}_{4}$ was diluted in $100 \mathrm{~mL}$ of water. From this dilution, $20 \mathrm{~mL}$ was used to dissolve $1.39 \mathrm{~g} \mathrm{FeSO}_{4} \cdot 7 \mathrm{H}_{2} \mathrm{O}$ $(0.025 \mathrm{M})$. Both solutions were degassed with $\mathrm{N}_{2}$ for $2 \mathrm{~h}$, while solution A was also stirred. After degassing, solution B was quickly added to solution A while stirring vigorously. After 10 min, stirring was stopped and the flask was placed in an oil bath that was preheated to $90{ }^{\circ} \mathrm{C}$ and left for $24 \mathrm{~h}$ under nitrogen. After heating, the oil bath was removed and the flask was left to cool down to room temperature. The nanoparticles were separated by magnetic decanting and washed until a stable dispersion was obtained, typically after 7 washing steps. The nanoparticles were finally dispersed in water.

Cobalt ferrite nanoparticles-The cobalt ferrite nanoparticles were prepared by the method reported by Tourinho et al. [42]: $2.39 \mathrm{~g}(10 \mathrm{mmol}) \mathrm{CoCl}_{2} \cdot 6 \mathrm{H}_{2} \mathrm{O}$ was dissolved in $5 \mathrm{~mL}$ of $2 \mathrm{M} \mathrm{HCl}$ and added to $5.48 \mathrm{~g} \mathrm{FeCl}_{3} \cdot 6 \mathrm{H}_{2} \mathrm{O}(20 \mathrm{mmol})$ dissolved in $40 \mathrm{~mL}$ of water. This mixture was briefly stirred and added to a boiling solution of $1 \mathrm{M} \mathrm{NaOH}(200 \mathrm{~mL})$ under vigorous stirring. Immediately a black/brown precipitate was formed and the mixture was heated to reflux for $1 \mathrm{~h}$. When cooled down to room temperature, the particles were magnetically sedimented and washed three times with $70 \mathrm{~mL}$ of water. The particles were redispersed in $50 \mathrm{~mL}$ of water and $30 \mathrm{~mL}$ of $2 \mathrm{M} \mathrm{HNO}_{3}$ was added. The mixture was vigorously stirred and $30 \mathrm{~mL}$ of $0.35 \mathrm{M} \mathrm{Fe}\left(\mathrm{NO}_{3}\right)_{3} \cdot 9 \mathrm{H}_{2} \mathrm{O}$ was added. The mixture was heated to reflux for $1 \mathrm{~h}$ and cooled down to room temperature. The particles were magnetically sedimented, washed once with water, and subsequently redispersed in Millipore water. 


\subsection{Positive Charging of Magnetic Nanoparticles}

For nanoparticle fixation to the hydrogel network, we found that the particles must be positively charged to facilitate spontaneous adsorption of acrylic acid. For this, approximately $200 \mathrm{mg}$ of nanoparticles of the original dispersion was taken. The particles were diluted with $500 \mathrm{~mL}$ of $2 \mathrm{M}$ $\mathrm{HNO}_{3}$ and stirred for $30 \mathrm{~min}$. Subsequently, the nanoparticles were washed several times until a stable dispersion in water was obtained. For magnetite (L) also ultrasonication was used to break up large aggregates present in the dispersion.

\subsection{Surface Modification of Magnetic Nanoparticles with Oleic Acid}

For some dissolution experiments, magnetite (S) nanoparticles were coated with oleic acid. In a typical experiment, approximately $200 \mathrm{mg}$ of charge stabilized magnetite nanoparticles were diluted with $250 \mathrm{~mL}$ water. This dispersion was stirred vigorously while oleic acid was added. When stirring was stopped, oleic acid formed a thick liquid phase on top of the water phase. If the water phase still contained nanoparticles, more oleic acid was added and the mixture was stirred again. This process was repeated until the water phase was white and turbid, typically after addition of $15 \mathrm{~mL}$ of oleic acid. The black top phase was washed with ethanol using a separation funnel, and the particles were finally dispersed in toluene or hexane.

\subsection{Preparation of Hydrogel and Fluid Samples for Magnetic Susceptibility Measurements}

For magnetic susceptibility measurements, two types of samples were used: a liquid dispersion of magnetic nanoparticles and magnetic nanoparticles incorporated in a hydrogel. For both types of sample, an equal amount of magnetic material was used. As initiator, V-50 was selected as there was no visible aggregation of the nanoparticles upon mixing with the monomers, in contrast to preliminary experiments with free radical polymerization using ammonium persulfate and $N, N, N^{\prime}, N^{\prime}$-tetramethylethylenediamine (TEMED). The monomer mixture composition was as follows: $0.33 \mathrm{~mL}$ of acrylic acid, $5.00 \mathrm{~mL}$ of hydroxyethyl acrylate, and $0.59 \mathrm{~mL}$ of diethylene glycol diacrylate. For the magnetite (S) dispersion sample, $0.8 \mathrm{~mL}$ of $20 \mathrm{mg} / \mathrm{mL}$ dispersion was diluted with $0.2 \mathrm{~mL}$ of water to obtain a $16 \mathrm{mg} / \mathrm{mL}$ dispersion. For the hydrogel sample, $0.8 \mathrm{~mL}$ of $20 \mathrm{mg} / \mathrm{mL}$ dispersion was diluted with $0.05 \mathrm{~mL}$ of water, $0.1 \mathrm{~mL}$ of monomer mixture, and $0.05 \mathrm{~mL}$ of initiator solution (V-50, $12 \mathrm{mg} / \mathrm{mL}$ ). The mixture was shaken and transferred to a measurement tube. The tube was put in an oven at $70{ }^{\circ} \mathrm{C}$ for polymerization for $15 \mathrm{~min}$. The sample preparation and total sample volume were similar for magnetite (L) and cobalt ferrite. For magnetite (L), $0.63 \mathrm{~mL}$ of a $32 \mathrm{mg} / \mathrm{mL}$ stock dispersion was used and for cobalt ferrite $0.5 \mathrm{~mL}$ of a $40 \mathrm{mg} / \mathrm{mL}$ stock dispersion was used.

\subsection{Characterization}

Transmission electron microscopy images were obtained from a Philips Tecnai 12 microscope operating at $120 \mathrm{kV}$. The particle size was determined by measuring at least 300 particles individually by hand using iTEM software (Olympus, Münster, Germany).

Infrared spectra were recorded on a Frontier FT-NIR/MIR Spectrometer from Perkin Elmer. Samples of positively charged particles for IR were prepared by adding a small amount of dispersion 
to $250 \mathrm{mg} \mathrm{KBr}$, with approximately $1 \mathrm{mg}$ of magnetic nanoparticles. The $\mathrm{KBr}$ samples were dried overnight at $80{ }^{\circ} \mathrm{C}$, ground to fine powder and pressed into pellets. Before measuring, the pellets were dried for $4 \mathrm{~h}$ at $80^{\circ} \mathrm{C}$ to remove water that was incorporated during preparation of the pellets. Samples of particles coated with acrylic acid were prepared by exposing the particles to a 1:1 mixture of acrylic acid and water for a few minutes. The particles were magnetically sedimented and washed three times with acetone. The particles dispersed in acetone were put on an ATR crystal and a measurement could be done when all acetone had evaporated.

Magnetization curves were measured at room temperature on a Micromag 2900 alternating gradient magnetometer from Princeton Measurement Corporation.

Frequency dependent magnetic susceptibility measurements were performed at $22{ }^{\circ} \mathrm{C}$ in the low-field limit $(H<170 \mathrm{~A} / \mathrm{m})$ on a homebuilt setup described by Kuipers et al. [43,44], whose $0.1 \mathrm{~Hz}-1 \mathrm{kHz}$ frequency range was extended to $1 \mathrm{MHz}$ using 7280 DSP lock-in amplifiers.

\section{Conclusions}

Hydrogels were prepared in which magnetic nanoparticles act effectively as cross-linkers. To examine the rotational mobility of the particles, our approach was to measure the low-field AC magnetic susceptibility in a frequency range that corresponds to Brownian rotation of single nanoparticles. The large decrease of the AC susceptibility of hydrogels with cobalt ferrite compared to the dispersion is due to its high magnetic anisotropy and the prohibition of Brownian relaxation in the hydrogel. The same is observed for iron oxide particles with a diameter of $23 \mathrm{~nm}$, whose magnetic particle moment is thermally blocked. Smaller iron oxide particles with a diameter around $9 \mathrm{~nm}$ exhibit fast Néel relaxation at frequencies of $1 \mathrm{MHz}$ and below, so that it does not matter whether the nanoparticles are fixed inside a hydrogel or dispersed in a liquid. However, the chemistry of the small iron oxide particles is the same as that of the larger ones, and we conclude that they also were irreversibly bound to the polymer network. This was expected a priori from our use of nanoparticles with adsorbed polymerizable surface species but now has been demonstrated by characterizing the rotational diffusion of the larger particles. A ferrohydrogel with frequency-independent response up to at least $1 \mathrm{MHz}$ appears suitable for in vivo biosensors of the type proposed by van Bruggen and van Zon, which was previously tested at $50 \mathrm{kHz}$ [7]. Currently, we are carrying out a systematic study of the swelling of our ferrohydrogels and how this can be detected magnetically.

\section{Acknowledgments}

This research is supported by the Dutch Technology Foundation STW, which is part of the Netherlands Organisation for Scientific Research (NWO), and which is partly funded by the Ministry of Economic Affairs. We thank Suzanne Verkleij and Rocio Costo Camara for helping to prepare magnetic nanoparticles. We also thank Bonny Kuipers and Emile Bakelaar for their help with the magnetic susceptibility setup.

\section{Conflict of Interest}

The authors declare no conflict of interest. 


\section{References}

1. Gupta, P.; Vermani, K.; Garg, S. Hydrogels: From controlled release to $\mathrm{pH}$-responsive drug delivery. Drug Discov. Today 2002, 7, 569-579.

2. Qin, J.; Asempah, I.; Laurent, S.; Fornara, A.; Muller, R.N.; Muhammed, M. Injectable superparamagnetic ferrogels for controlled release of hydrophobic drugs. Adv. Mater. 2009, 21, 1354-1357.

3. Pankhurst, Q.; Connolly, J.; Jones, S.; Dobson, J. Applications of magnetic nanoparticles in biomedicine. J. Phys. D Appl. Phys. 2003, 36, R167-R181.

4. Han, I.; Han, M.; Kim, J.; Lew, S.; Lee, Y.; Horkay, F.; Magda, J. Constant-volume hydrogel osmometer: A new device concept for miniature biosensors. Biomacromolecules 2002, 3, 1271-1275.

5. Urban, G.A.; Weiss, T. Hydrogels for Biosensors. In Hydrogel Sensors and Actuators: Engineering and Technology, 2010 ed.; Gerlach, G., Arndt, K.-F., Eds.; Springer: Berlin, Germany, 2009; pp. 197-220.

6. Van der Linden, H.; Herber, S.; Olthuis, W.; Bergveld, P. Stimulus-sensitive hydrogels and their applications in chemical (micro)analysis. Analyst 2003, 128, 325-331.

7. Van Bruggen, M.P.B.; van Zon, J.B.A. Theoretical description of a responsive magneto-hydrogel transduction principle. Sens. Actuators A Phys. 2010, 158, 240-248.

8. Ilg, P. Stimuli-responsive hydrogels cross-linked by magnetic nanoparticles. Soft Matter 2013, 9, 3465-3468.

9. Messing, R.; Frickel, N.; Belkoura, L.; Strey, R.; Rahn, H.; Odenbach, S.; Schmidt, A.M. Cobalt ferrite nanoparticles as multifunctional cross-linkers in PAAm ferrohydrogels. Macromolecules 2011, 44, 2990-2999.

10. Vo, D.Q.; Kim, E.; Kim, S. Surface modification of hydrophobic nanocrystals using short-chain carboxylic acids. J. Colloid Interface Sci. 2009, 337, 75-80.

11. Astalan, A.; Ahrentorp, F.; Johansson, C.; Larsson, K.; Krozer, A. Biomolecular reactions studied using changes in Brownian rotation dynamics of magnetic particles. Biosens. Bioelectron. 2004, 19, 945-951.

12. Chung, S.; Hoffmann, A.; Bader, S.; Liu, C.; Kay, B.; Makowski, L.; Chen, L. Biological sensors based on Brownian relaxation of magnetic nanoparticles. Appl. Phys. Lett. 2004, 85, 2971-2973.

13. Kotitz, R.; Weitschies, W.; Trahms, L.; Brewer, W.; Semmler, W. Determination of the binding reaction between avidin and biotin by relaxation measurements of magnetic nanoparticles. J. Magn. Magn. Mater. 1999, 194, 62-68.

14. Fornara, A.; Johansson, P.; Petersson, K.; Gustafsson, S.; Qin, J.; Olsson, E.; Ilver, D.; Krozer, A.; Muhammed, M.; Johansson, C. Tailored magnetic nanoparticles for direct and sensitive detection of biomolecules in biological samples. Nano Lett. 2008, 8, 3423-3428.

15. Hong, C.; Wu, C.; Chiu, Y.; Yang, S.; Horng, H.; Yang, H. Magnetic susceptibility reduction method for magnetically labeled immunoassay. Appl. Phys. Lett. 2006, 88, 212512:1-212512:3.

16. Barrera, C.; Florian-Algarin, V.; Acevedo, A.; Rinaldi, C. Monitoring gelation using magnetic nanoparticles. Soft Matter 2010, 6, 3662-3668.

17. Erne, B.H.; Butter, K.; Kuipers, B.W.M.; Vroege, G.J. Rotational diffusion in iron ferrofluids. Langmuir 2003, 19, 8218-8225. 
18. Fannin, P. Wideband measurement and analysis techniques for the determination of the frequency-dependent, complex susceptibility of magnetic fluids. Adv. Chem. Phys. 1998, 104, 181-292.

19. Rosensweig, R. Heating magnetic fluid with alternating magnetic field. J. Magn. Magn. Mater. 2002, 252, 370-374.

20. Chikazumi, S.; Charap, S.H. Physics of Magnetism, 1st ed.; John Wiley \& Sons, Inc: New York, NY, USA, 1964; p. 554.

21. Luigjes, B.; Woudenberg, S.M.C.; de Groot, R.; Meeldijk, J.D.; Galvis, H.M.T.; de Jong, K.P.; Philipse, A.P.; Erne, B.H. Diverging geometric and magnetic size distributions of iron oxide nanocrystals. J. Phys. Chem. C 2011, 115, 14598-14605.

22. Kodama, R. Magnetic nanoparticles. J. Magn. Magn. Mater. 1999, 200, 359-372.

23. Batlle, X.; Labarta, A. Finite-size effects in fine particles: Magnetic and transport properties. J. Phys. D Appl. Phys. 2002, 35, R15-R42.

24. Barker, A.; Cage, B.; Russek, S.; Stoldt, C. Ripening during magnetite nanoparticle synthesis: Resulting interfacial defects and magnetic properties. J. Appl. Phys. 2005, 98, 063528:1-063528:7.

25. Goya, G.; Berquo, T.; Fonseca, F.; Morales, M. Static and dynamic magnetic properties of spherical magnetite nanoparticles. J. Appl. Phys. 2003, 94, 3520-3528.

26. Roca, A.G.; Morales, M.P.; O’Grady, K.; Serna, C.J. Structural and magnetic properties of uniform magnetite nanoparticles prepared by high temperature decomposition of organic precursors. Nanotechnology 2006, 17, 2783-2788.

27. Tartaj, P.; Morales, M.D.; Veintemillas-Verdaguer, S.; Gonzalez-Carreno, T.; Serna, C.J. The preparation of magnetic nanoparticles for applications in biomedicine. J. Phys. D Appl. Phys. 2003, 36, R182-R197.

28. Zhao, L.; Zhang, H.; Xing, Y.; Song, S.; Yu, S.; Shi, W.; Guo, X.; Yang, J.; Lei, Y.; Cao, F. Studies on the magnetism of cobalt ferrite nanocrystals synthesized by hydrothermal method. J. Solid State Chem. 2008, 181, 245-252.

29. Tang, C.; Wang, C.; Chien, S. Characterization of cobalt oxides studied by FT-IR, Raman, TPR and TG-MS. Thermochimica Acta 2008, 473, 68-73.

30. Cornell, R.M.; Schwertmann, U. The Iron Oxides; VCH Verlagsgesellschaft: Weinheim, Germany, 1996; p. 573.

31. Max, J.; Chapados, C. Infrared spectroscopy of aqueous carboxylic acids: Comparison between different acids and their salts. J. Phys. Chem. A 2004, 108, 3324-3337.

32. Klokkenburg, M.; Hilhorst, J.; Erne, B.H. Surface analysis of magnetite nanoparticles in cyclohexane solutions of oleic acid and oleylamine. Vib. Spectrosc. 2007, 43, 243-248.

33. Pretsch, E.; Buhlmann, P.; Badertscher, M. Structure Determination of Organic Compounds; Springer-Verlag: Berlin, Germany, 2009.

34. Klokkenburg, M.; Dullens, R.; Kegel, W.; Erne, B.; Philipse, A. Quantitative real-space analysis of self-assembled structures of magnetic dipolar colloids. Phys. Rev. Lett. 2006, 96, 037203:1-037203:4.

35. Klokkenburg, M.; Erne, B.H.; Wiedenmann, A.; Petukhov, A.V.; Philipse, A.P. Dipolar structures in magnetite ferrofluids studied with small-angle neutron scattering with and without applied magnetic field. Phys. Rev. E 2007, 75, 051408. 
36. Klokkenburg, M.; Erne, B.H. Comparison of reversible and irreversible dipolar assemblies in a ferrofluid. J. Magn. Magn. Mater. 2006, 306, 85-91.

37. Klokkenburg, M.; Erne, B.H.; Philipse, A.P. Thermal motion of magnetic iron nanoparticles in a frozen solvent. Langmuir 2005, 21, 1187-1191.

38. Galicia, J.A.; Cousin, F.; Dubois, E.; Sandre, O.; Cabuil, V.; Perzynski, R. Static and dynamic structural probing of swollen polyacrylamide ferrogels. Soft Matter 2009, 5, 2614-2624.

39. Galicia, J.A.; Cousin, F.; Dubois, E.; Sandre, O.; Cabuil, V.; Perzynski, R. Local structure of polymeric ferrogels. J. Magn. Magn. Mater. 2011, 323, 1211-1215.

40. Massart, R. Preparation of aqueous magnetic liquids in alkaline and acidic media. IEEE Trans. Magn. 1981, 17, 1247-1248.

41. Andres Verges, M.; Costo, R.; Roca, A.G.; Marco, J.F.; Goya, G.F.; Serna, C.J.; Morales, M.P. Uniform and water stable magnetite nanoparticles with diameters around the monodomain-multidomain limit. J. Phys. D Appl. Phys. 2008, 41, 134003.

42. Tourinho, F.; Franck, R.; Massart, R. Aqueous ferrofluids based on manganese and cobalt ferrites. J. Mater. Sci. 1990, 25, 3249-3254.

43. Kuipers, B.W.M.; Bakelaar, I.A.; Klokkenburg, M.; Erne, B.H. Complex magnetic susceptibility setup for spectroscopy in the extremely low-frequency range. Rev. Sci. Instrum. 2008, 79, 013901.

44. Erne, B.H.; Claesson, M.; Sacanna, S.; Klokkenburg, M.; Bakelaar, E.; Kuipers, B.W.M. Low-Frequency complex magnetic susceptibility of magnetic composite microspheres in colloidal dispersion. J. Magn. Magn. Mater. 2007, 311, 145-149.

(C) 2013 by the authors; licensee MDPI, Basel, Switzerland. This article is an open access article distributed under the terms and conditions of the Creative Commons Attribution license (http://creativecommons.org/licenses/by/3.0/). 